7 - ORIGINAL ARTICLE

ISCHEMIA-REPERFUSION

\title{
Direct intraperitoneal resuscitation with lidocaine, methylene blue and pentoxiphylline combination does not decreases inflammation after intestinal ischemia-reperfusion injury in rats ${ }^{1}$
}

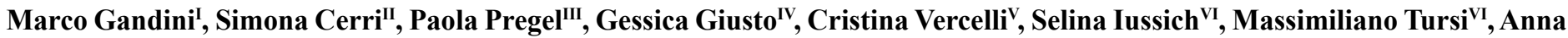 \\ Maria Farca ${ }^{\text {II }}$ \\ DOI: http://dx.doi.org/10.1590/S0102-865020160050000007

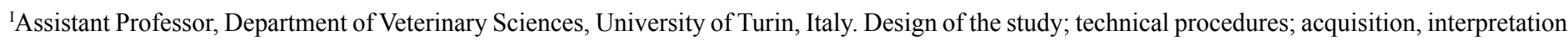 \\ and analysis of data; manuscript preparation and writing. \\ IIDVM, Department of Clinical Sciences, Faculty of Veterinary Medicine, University of Liege, Belgium. Technical procedures; acquisition, interpretation \\ and analysis of data; manuscript preparation and writing.

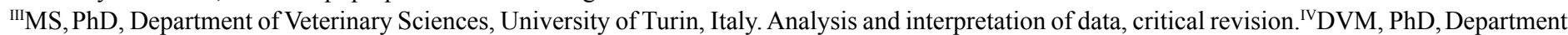 \\ of Veterinary Sciences, University of Turin, Italy. Manuscript writing, critical revision. ${ }^{\mathrm{V}} \mathrm{MS}, \mathrm{PhD}$, Department of Veterinary Sciences, University of \\ Turin, Italy. Interpretation and analysis of data, manuscript preparation and writing. \\ ${ }^{\mathrm{V}}$ Assistant Professor, Department of Veterinary Sciences, University of Turin, Italy. Histopathological examination, critical revision. ${ }^{\mathrm{VII}}$ Full Professor, \\ Department of Veterinary Sciences, University of Turin, Italy. Design of the study, interpretation and analysis of data, manuscript preparation and \\ writing.
}

\footnotetext{
ABSTRACT

PURPOSE: To evaluate the effects of an intraperitoneal solution of methylene blue (MB), lidocaine and pentoxyphylline (PTX) on intestinal ischemic and reperfusion injury

METHODS: Superior mesenteric artery was isolated and clamped in 36 adult male Sprague Dawley rats. After 60 minutes, clamp was removed and a group received intraperitoneally UNITO solution (PTX 25mg/kg + lidocaine $5 \mathrm{mg} / \mathrm{kg}+\mathrm{MB} 2 \mathrm{mg} / \mathrm{kg}$ ), while the other group was treated with warm $0.9 \% \mathrm{NaCl}$ solution. Rats were euthanized $45 \mathrm{~min}$ after drug administration. Lung and bowel were collected for histological evaluation (using Park's score) and determination of myeloperoxidase (MPO) and malondialdehyde (MDA) levels.

RESULTS: Control samples showed lymphoplasmocytic infiltrate and crypt necrosis of villi. MPO and MDA measurements shown no differences between treated and control groups.

CONCLUSION: The combination of lidocaine, methylene blue and pentoxyphylline administered intraperitoneally at the studied dose, did not decreased histological lesion scores and biochemical markers levels in intestinal ischemia/reperfusion injury.

Key words: Ischemia. Reperfusion. Lung. Histology. Rats.
} 


\section{Introduction}

Intestinal $\mathrm{I} / \mathrm{R}$ injury is a common pathologic event associated with many intestinal pathologies, whenever the intestine undergoes a reduction with subsequent restoration (usually during surgery) of blood flow ${ }^{1-5}$. Spontaneous I/R can occur in humans and if the diagnosis is made within $24 \mathrm{~h}$ after the onset of symptoms and aggressive treatment initiated, acute mesenteric ischemia has about a $50 \%$ survival rate, whereas this rate drops to $30 \%$ or less when diagnosis is delayed ${ }^{6}$. In experimental models, it has been shown that the extent of mucosal damage is a direct function of time elapsed from the onset of mesenteric artery occlusion with first histological changes after $30 \mathrm{~min}$ and more prominent destruction of the villi after $60 \mathrm{~min}^{7}$. After revascularization, mucosal regeneration via cell migration occurs rapidly, even after 90 min of ischemia ${ }^{8}$.

Several authors investigated the $\mathrm{I} / \mathrm{R}$ phenomenon and tried therapeutic approaches but in the majority of these it was not possible to identify a single compound completely capable to prevent and solve I/R damages, but the combination of some drugs seemed to suggest a synergic effect that enhance protection, acting on different phases of this process ${ }^{5}$.

Pentoxifylline (PTX), a methylxanthine derivative known for many years for its rheological properties, has proven to be a potent inhibitor of tumor necrosis factor (TNF) production. It has been suggested that PTX can enhance the chemotactic response of neutrophils, but may inhibit phagocytosis and the superoxide production by neutrophils and monocytes. It has also been shown that this drug has beneficial effects in patients with lung I/R injury. The potential local effects are important for acute lung injury manifestation by inflammatory mediators that are blood- and lymph-borne, and oxygen-free radical activations ${ }^{10}$.

Methylene blue (MB) is an inexpensive drug used to treat diseases such as methaemoglobinemia and Alzheimer's and has been proposed for use in vasoplegic shock during sepsis, cardiopulmonary bypass surgery and liver transplantation. However, MB is known to enhance key biochemical pathways in mithocondria, and cycling between oxidized and reduced $\mathrm{MB}$ forms might block oxidant production ${ }^{11}$.

Lidocaine is widely used as a local anesthetic, but has been recently administered systemically in horses to treat post operative ileus. It is known that local anesthetic agents modulate the inflammatory response via mechanisms unrelated to sodium channel blockade. Lidocaine has been shown to reduce cytokines release and inhibit neutrophil function. Additionally, in studies evaluating its effect on I/R injury in other organs, lidocaine has been shown to reduce lipid peroxidation attributable to oxidant release and inhibit neutrophil adhesion and migration ${ }^{12}$. It has been also demonstrated to inhibit neutrophils migration ${ }^{13}$.

Taking into consideration the previous studies, it was hypothesized that a mixture of pentoxifylline, lidocaine and methylene blue could be useful in prevention and treatment of intestinal $\mathrm{I} / \mathrm{R}$ injury. For this purpose we used an animal model of intestinal $\mathrm{I} / \mathrm{R}$ in which the superior mesenteric artery occlusionreperfusion simulates the $\mathrm{I} / \mathrm{R}$ injury that may occur in clinical situations, and investigated the effects of the combination of drugs on bowel and lungs.

\section{Methods}

The study was approved by the Bioethical committee of the University of Turin and by the Italian Ministry of Health.

Thirty-six Sprague-Dawley adult male rats, body weights of 300 grams (270-310 grams), were kept in standard conditions and fed standard rat chow for 5 days before the experiment. Twelve hours before surgery they were fasted but water allowed ad libitum. Anesthesia was induced by a single intramuscular injection of xylazine $(0.025 \mathrm{ml} / \mathrm{kg})$ and ketamine $(25 \mathrm{mg} / \mathrm{rat})$, and the abdomen shaved and aseptically prepared with $10 \%$ povidone iodine. Upon a 2.5-cm midline laparotomy, the superior mesenteric artery was isolated at its origin and occluded with an atraumatic microvascular clamp for $60 \mathrm{~min}$. To ensure a complete ischemia, collateral arteries were blocked using an encircling ligature with Prolene USP 4-0 suture. At the end of ischemia period the clamp and ligatures were removed and reperfusion was confirmed by restoration of the pulsations and the normal gut color. The animals were randomly assigned with a random calculator (www.random. org) to two groups: treated (T) and control (C). In group T, 18 rats were treated with $1 \mathrm{ml}$ of the solution (UNITO solution) composed by PTX $(25 \mathrm{mg} / \mathrm{kg})$, lidocaine $(5 \mathrm{mg} / \mathrm{kg})$ and $\mathrm{MB}$ $(6 \mathrm{mg} / \mathrm{kg}$ ) diluted in warm $0.9 \% \mathrm{NaCl}$ solution administered intraperitoneally. In group C, 18 rats were administered $1 \mathrm{ml}$ of warm $0.9 \% \mathrm{NaCl}$ solution intraperitoneally. Experiments were made in 6 different sessions involving 3 rats per each group. After 45 min of reperfusion, rats were euthanized by cervical vertebrae dislocation.

\section{Samples collection}

Lungs and bowel samples were collected and stored in formalin or at $-80^{\circ} \mathrm{C}$ for biochemical analysis.Histology 
Samples were fixed in buffered formalin, embedded in paraffin, and 4- $\mu \mathrm{m}$ tissue sections were stained with hematoxylineosin (HE). The slides were independently evaluated by two histopathologists blinded to the group assignation. To evaluate tissue damages, Park's score was used ${ }^{14}$ (Chart 1).

CHART 1 - Description of the histological scoring system after Park and Chiu ${ }^{8}$.

\begin{tabular}{lc}
\hline 0 & Normal mucosa \\
1 & Subepithelial space at villus tips \\
2 & Extension of subepithelial space with moderate lifting \\
3 & Massive lifting down sides of villi, some denuded tips \\
4 & Denuded villi, dilated capillaries \\
5 & Disintegration of lamina propria \\
6 & Crypt layer injury \\
7 & Transmucosal infarction \\
8 & Transmural infarction \\
\hline
\end{tabular}

\section{Biochemical analysis}

The biochemical analysis consisted in myeloperoxidase (MPO) and malondialdehyde (MDA) activity evaluation according, respectively, to the methods proposed by Muià et al. ${ }^{15}$ and Hei et $a l .{ }^{16}$, but introducing some minor modifications.

\section{MPO}

MPO activity was measured both in lung and intestine. Aliquots of $500 \mathrm{mg}$ of frozen tissues were homogenated in potassium phosphate buffer (PPB) $(10 \mathrm{mM}$ and $\mathrm{pH} 7)$ and $0.5 \%(\mathrm{w} / \mathrm{v})$ hexadecyltrimethyl-ammonium bromide (CTAB, Sigma Aldrich, Italy), and centrifuged at $20.000 \mathrm{x} g$ for $30 \mathrm{~min}$ at $4^{\circ} \mathrm{C}$. Fifty $\mu \mathrm{L}$ of surnatant were incubated with $950 \mu \mathrm{L}$ of tetramethylbenzidine (TMB plus, Kem-EN-Ten Diagostic, Denmark) and rate of change in absorbance was measured at $650 \mathrm{~nm}$.

\section{$M D A$}

MDA content was measured in intestine samples. Tissues homogenate $(50 \mu \mathrm{L})$ were incubated with $50 \mu \mathrm{L} 8.1 \%$ sodium dodecyl sulfate (SDS, Sigma Aldrich, Italy), $400 \mu \mathrm{L}$ acetic acid buffer (Sigma Aldrich, Italy), $400 \mu \mathrm{L} 0.8 \%$ thiobarbituric acid (TBA, Sigma Aldrich, Italy) and $100 \mu \mathrm{L}$ distilled water.

Aliquots of of 1,1,3,3-tetraethoxypropane (Sigma Aldrich, Italy) were used as positive control.
Then all the tubes were incubated at $95^{\circ} \mathrm{C}$ for $1 \mathrm{~h}$. After cooled at -20 for $5 \mathrm{~min}, 1 \mathrm{~mL}$-butyl alcohol (Sigma Aldrich, Italy) was added into the sample, which was centrifuged for 10 $\min$ at $800 \mathrm{x} g$. The supernatant of the samples was taken to detect absorbance at $533 \mathrm{~nm}$ with spectrophotometer. To determine the concentration we used a molar extinction coefficient for MDA of $1.56 \times 10^{5} \mathrm{~cm}^{-1} \mathrm{M}^{-1}$. Results were expressed as nmol MDA/100mg $\operatorname{protein}^{17}$.

\section{Statistical analysis}

All data are presented as mean \pm standard error (SEM). Normality of data was assessed with the Kolmogornov-Smirnoff test. Differences for histological score comparison were analyzed with a Mann Whitney test. Unpaired T-test was performed to analyze biochemical results. Statistical analyses were performed by GraphPad InStat (vers. 3.05) statistical software (GraphPad Inc.). The Grubbs test was used to reveal potential outliers, which were excluded. A value of $\mathrm{p}<0.05$ was considered to be statistically significant.

\section{Results}

During the experiment, two rats of treated group died during recovery from anesthesia.

\section{Histology}

Histological score for the control group was $3.4 \pm 0.6817$ while in the treated group was $2.47 \pm 0.6537$. The difference was not statistically significant $(\mathrm{p}=0.3242)$.

Malondialdehyde level in the intestine was not significantly different $(\mathrm{p}=0.2884)$ between control $(88.3 \pm 27.234$ $\mathrm{nmol} / 100 \mathrm{mg})$ and treated I/R group $(80.28 \pm 14.802 \mathrm{nmol} / 100$ $\mathrm{mg})$.

The MPO activity in the intestine did not differ significantly $(\mathrm{p}=0.6834)$ between treated $(1.156 \pm 0.35 \mathrm{mU} / \mathrm{g})$ and control group $(1.349 \pm 0.39 \mathrm{mU} / \mathrm{g})$.

The MPO activity in the lung was not significantly different $(\mathrm{p}=0.5686)$ between control $(3.06 \pm 0.497 \mathrm{mU} / \mathrm{g})$ and treated I/R group $(3.235 \pm 0.583 \mathrm{mU} / \mathrm{g})$.

\section{Discussion}

The results of the present study highlighted that the UNITO solution (mixture of PXT, MB and lidocaine) is not 
effective against I/R injuries when administered intraperitoneally. Analysis of the data showed that MPO and MDA increased in all the tested samples without significant differences between groups.

In the authors' best knowledge, this was the first time that this combination was used. The authors' hypothesis was that these substances could have a synergic effect on I/R bowel and lung injuries. This hypothesis was supported by the bibliography that showed a huge number of papers dealing with the experimental success of single molecule trial, suggesting a further clinical application.

The study of Marqui et al. ${ }^{10}$ demonstrated that pentoxifylline has preventive effects and therapeutic potential in I/R-induced lung injuries, but the same authors are carefull to suggest a direct application while the clinical relevance of that manuscript referred to the use of pentoxifylline in situations requiring procedures of ischemia with reperfusion to reduce or prevent distant organs damage. The study of Seifi et al. ${ }^{18}$ showed that PTX and N- Acetilcysteine (NAC) administered intraperitoneally protected kidney tissue against oxidative damage caused by experimental induced hepatic I/R. Kalay et $a l .{ }^{19}$ and Lloris Carsi et al. ${ }^{1}$ demonstrated the beneficial effects of intraperitoneally administered pentoxyphilline on intestinal injury after ischemic and reperfusion event in an in vivo animal model.

The study of Salehi et al. ${ }^{20}$ demonstrate that a treatment with a amino acid-based (AA) solution could reduce neutrophil infiltration in tissues reperfused after $30 \mathrm{~min}$ of ischemic event, indicating the possibility of lower graft immunogenicity over this time frame. Presumably, reduced neutrophil localization implies a decrease of chemotactic factors and influencing reduction of neutrophils attraction to the site of injury. Although diminished neutrophil recruitment supports the link between I/R injury and inflammatory response, additional adjunctive therapies involving anti-inflammatory agents seem to be required to fully protect the injured mucosa during inflammatory attack following I/R. The study of Berger et al..$^{13}$ demonstrated that systemic administration of lidocaine arrest transmigration of neutrophils through endothelial cells and the results obtained by Elhakim et al. ${ }^{21}$ demonstrated that intraperitoneal lidocaine enhance bowel recovery after surgery.

First in 1988, Kelner et al..$^{22}$, reviewing the mechanisms of action of methylene blue, observed that it could be used to inhibit the production of superoxides in ischemic tissues. For the same reason this molecule was chosen in other experiments ${ }^{23-26}$ and in the present study. On the other side, the study of Miranda et al. ${ }^{27}$ demonstrated that MB infusion had not beneficial effect on the healing of intestinal anastomoses in an experimental I/R model in rats.
Seen these previous works we hypothesized that a combination of these molecules could have a synergic effect on different aspects of $\mathrm{I} / \mathrm{R}$ injuries in intestines and lungs. The scarce efficacy of the mixture could be due to different causes.

First, the dosage of each component in the mixture has been extrapolated from previous literature. By testing other doses of each components, results could be different.

Second, the intraperitoneal administration may be effective for some of the components, but not for others. Lidocaine, for instance, has been extensively tested for intravenous administration, and very little for intraperitoneal administration. Although intraperitoneal absorption can be comparable to intravenous absorption for some drugs, this could be not the case for lidocaine.

Third, I/R injury involves complex pathways and many of these could not be targeted by the intraperitoneal route. Last possibility is that instead of having a synergic effect, the combination of these molecules has a counter effective effect on the efficacy of all or some of them.

Further studies should be conducted to evaluate these limits, considering different dosages and different routes of administration.

\section{Conclusion}

The combination of lidocaine, methylene blue and pentoxyphylline administered intraperitoneally at the dose hereby presented, did not significantly decreased histological lesion scores and biochemical markers levels in intestinal ischemia/reperfusion injury.

\section{References}

1. Lloris Carsi JM, Cejalvo Lapeña D, Toledo AH, Zaragoza Fernandez C, Toledo Pereyra LH. Pentoxifylline protects the small intestine after severe ischemia and reperfusion. Exp Clin Tranplant. 2013;11(3):250-258. PMID: 23767943.

2. Carden DL, Granger DN. Pathophysiology of ischaemia-reperfusion injury. J Pathol. 2000;190:255--266. PMID: 10685060.

3. Devarajan P. Update on mechanisms of ischemic acute kidney injury. J Am Soc Nephrol. 2006;17:1503-1520. PMID: 16707563.

4. Perico N, Cattaneo D, Sayegh MH, Remuzzi G. Delayed graft function in kidney transplantation. Lancet. 2004;364:1814-1827. PMID: 15541456.

5. Ilhan H, Alatas O, Tokar B, Colak O, Pasaoglu O, Koku N. Effects of the Anti-ICAM-1 Monoclonal antibody, allopurinol, and methylene blue on intestinal reperfusion injury. J Pediatric Surg. 2003;38:15911595. PMID: 14614706.

6. Kassahun WT, Schulz T, Richter O, Hauss J. Unchanged high mortality rates from acute occlusive intestinal ischemia: six year review. Langenbecks Arch Surg. 2008;393:163-171. PMID: 18172675 . 
7. Ikeda H, Suzuki Y, Suzuki M, Kike M, Tamura J, Tong J, Nomura M, Itoh G. Apoptosis is a major mode of cell death caused by ischaemia and ischaemia/reperfusion injury to the rat intestinal epithelium. Gut. 1998;42:530-537. PMID: 9616316.

8. Quaedackers JSLT, Beuk RJ, Bennet L, Charlton A, oude Egbrink MG, Gunn AJ, Heineman E. An evaluation of methods for grading histologic injury following ischemia/reperfusion of the small bowel. Transplant Proc. 2000;32:1307-1310. PMID: 10995960.

9. Kalogeris T, Baines CP, Krenz M, Korthuis RJ. Cell biology of ischemia/reperfusion injury. Int Rev Cell Mol Biol. 2012; 298:229 317. PMID: 22878108.

10. Marqui CE, Silva HCA, Ferez DC, Cavassan SS, Moraes JB, Silva DA, Simoes RS, Lopes CA, Taha MO, Oliveira-Junior IS. Pretreatment with pentoxifylline attenuates lung injury induced by intestinal ischemia/reperfusion in rats. Acta Cir Bras. 2011;26: 438444. PMID: 22042105.

11. Collange OL, Charles AL, Bouitbir J, Chenard MP, Zoll J, Diemunsch P, Thaveau F, Chakfè N, Piquard F, Geny B. Methylene blue protects liver oxidative capacity after gut ischaemia-reperfusion in the rat Eur J Vasc Endovasc Surg. 2013;45(2):168-75. PMID: 23246335.

12. Cook VL, Jones SJ, McDowell M, Campbell NB, Davis JL, Blikslager AT. Attenuation of ischaemic injury in the equine jejunum by administration of systemic lidocaine. Eq Vet J. 2008;40(4), 353357. PMID: 18321812.

13. Berger C, Rossaint J, Van Aken H, Westphal M, Hahnenkamp K, Zarbock A. Lidocaine reduces neutrophil recruitment by abolishing chemokine-induced arrest and transendothelial migration in septic patients. J of Immunol. 2014;192:367-376. PMID: 24293632.

14. Park PO, Haglund U. Regeneration of small bowel mucosa after intestinal ischemia. Crit Care Med. 1992; 20:135-139. PMID: 1729031

15. Muià C, Mazzon E, Di Paola E, Genovese T, Menegazzi M, Caputi AP, Suzuki H, Cuzzocrea S. Green tea polyphenol extract attenuates ischemia/reperfusion injury of the gut. Naunyn-Schmiedeberg's Arch Pharmacol. 2005;371:364-374. PMID: 15997392.

16. Hei ZQ, Huang HQ, Zhang JJ, Chen BX, Li XY. Protective effect of Astragalus membranaceus on intestinal mucosa reperfusion injury after hemorrhagic shock in rats. World $\mathrm{J}$ Gastroenterol. 2005;11(32):4986-4991. PMID: 161224050.

17. Uslu C, Taysi S, Bakan N. Lipid peroxidation and antioxidant enzyme activities in experimental maxillary sinusitis. Ann Clin Lab Sci. 2003; 33: 18-22. PMID: 12661894.

18. Seifi B, Kadkhodaee M, Delevari F, Mikaeili S, Shams S, Ostad $\mathrm{SN}$. Pretreatment with pentoxifylline and $\mathrm{N}$-acetylcysteine in liver ischemia reperfusion-induced renal injury. Renal Fail. 2012;34(5):610-615. PMID: 22364443.

19. Kalay S, Islek A, Ozturk A, Kalay Z, Elpek O, Aldemir H, Akcakus M, Oygur N. Pentoxifylline therapy attenuates intestinal injury in rat pups with hypoxic ischemic encephalopathy. J Matern Fetal Neonatal Med. 2014;27(14):1476-1480. PMID: 24195683.

20. Salehi P, Madsen K, Zhu J, Castillo E, Avilla J, Lakey JR, Churchill TA. Alleviating ischemia-reperfusion injury in small bowel. Am J of Transplant. 2004;4(5):728-737. PMID: 15084167.

21. Elhakim M, Amine H, Kamel S, Saad F. Effects of intraperitoneal lidocaine combined with intravenous or intraperitoneal tenoxicam on pain relief and bowel recovery after laparoscopic cholecystectomy. Acta Anaesthesiol Scand. 2000;44(8):929-933. PMID: 10981568.

22. Kelner MJ, Bagnell R, Hale B, Alexander NM. Methylene blue competes with paraquat for reduction by flavo-enzimes resulting in decreased superoxide production in the presence of hemeproteins. Arch Biochem Biophys. 1988;262(2):422-426. PMID: 2835006.
23. Heydrick SJ, Reed KL, Cohen Pa, Aarons CB, Gower AC, Becker $\mathrm{JM}$, Stucchi AF. Intraperitoneal administration of methylene blue attenuates oxidative stress, increases peritoneal fibrinolysis, and inhibits intraabdominal adhesion formation. J Surg Res. 2007;143(2):311-319. PMID: 17826794.

24. Weinbroum AA. Methylene blue attenuates lung injury after mesenteric artery clamping/unclamping. Eur $\mathrm{J}$ of Clin Invest. 2004;34:436-442. PMID: 15200496.

25. Weinbroum AA, Goldin I, Kluger Y, Szold A. Methylene blue in preventing hemodynamic and metabolic derangement following superior mesenteric artery clamping/unclamping: an intratracheal vs. intraperitoneal dose-response study. Shock. 2002;17(5):372-375. PMID: 12022756.

26. Galili Y, Ben-Abraham R, Weinbroum A, Marmur S, Iaina A, Volman Y, Peer G, Szold O, Soffer D, Klausner J, Rabau M, Kluger Y. Methylene blue prevents pulmonary injury following intestinal ischemia reperfusion. J Trauma. 1998;45:222-226. PMID: 9715176.

27. Miranda, EF, Greca FH, Noronha L, Kotze LR, Rubin MR. The influence of methylene blue on the healing of intestinal anastomoses subjected to ischemia and reperfusion in rats. Acta Cir Bras. 2010;25(1):63-70. PMID: 20126891.

\section{Correspondence:}

Dr. Marco Gandini

Department of Veterinary Sciences, University of Turin

Largo P. Braccini 2-5. Grugliasco (To) Italy

Phone: +390116708861

marco.gandini@unito.it

Received: Jan 22, 2016

Review: Mar 19, 2016

Accepted: Apr 18, 2016

Conflict of interest: none

Financial source: none

${ }^{1}$ Research performed at Department of Veterinary Sciences, University of Turin, Italy. 